\title{
INVESTIGATION OF CRACK RESISTANCE IN EPOXY/BORON NITRIDE NANOTUBE NANOCOMPOSITES BASED ON MULTI-SCALE METHOD
}

\author{
Hossein Hemmatian \\ Department of Mechanical Engineering, Semnan Branch, Islamic Azad University, Semnan, Iran \\ e-mail: hoseinhemmatian@gmail.com \\ Mohammad ReZA Zamani, JAFAR EskAndARI JAM \\ Faculty of Mechanical Engineering, Malek-Ashtar University of Technology, Tehran, Iran
}

\begin{abstract}
Boron nitride nanotubes (BNNTs) possess superior mechanical, thermal and electrical properties and are also suitable for biocomposites. These properties make them a favorable reinforcement for nanocomposites. Since experimental studies on nanocomposites are timeconsuming, costly, and require accurate implementation, finite element analysis is used for nanocomposite modeling. In this work, a representative volume element (RVE) of epoxy/BNNT nanocomposites based on multi-scale modeling is considered. The bonds of BNNT are modeled by $3 \mathrm{D}$ beam elements. Also non-linear spring elements are employed to simulate the van der Waals bonds between the nanotube and matrix based on the Lennard-Jones potential. Young's and shear modulus of BNNTs are in ranges of 1.039-1.041 TPa and 0.44-0.52 TPa, respectively. Three fracture modes (opening, shearing, and tearing) have been simulated and stress intensity factors have been determined for a pure matrix and nanocomposite by $J$ integral. Numerical results indicate that by incorporation of BNNT in the epoxy matrix, stress intensity factors of three modes decrease. Also, by increasing the chirality of BNNT, crack resistance of shearing and tearing modes are enhanced, and stress intensity factor of opening mode reduced. BNNTs bridge the crack surface and prevent crack propagation.
\end{abstract}

Keywords: boron nitride nanotube, epoxy, fracture modes, finite element model, multi-scale method

\section{Introduction}

Nanostructures as a new class of materials are prevalently used in the recent years. One of the most commonly used nanostructure is carbon nanotube (CNT), and one similar structure newer than CNTs is boron nitride nanotube (BNNT) (Chopra et al., 1995). BNNTs, like CNTs, have extraordinary mechanical properties (Chopra and Zettl, 1998), high thermal conductivity (Chang et al., 2005), and good resistance against oxidation at high temperature (Chen et al., 2004). Despite their similar structures, BNNTs have different properties because BNNTs are composed of various atoms (Fereidoon et al., 2015). Metal, semiconductor or insulator characteristics of CNTs are highly depending on chirality, diameter, and number of walls, while BNNTs behave independently as an insulator for low electric fields (Khaleghian and Azarakhshi, 2016; Molani 2017). BNNTs are also found to be nontoxic to health and environment due to their chemical inertness and structural stability. Therefore, BNNT is particularly suitable for biological applications.

The elastic properties of BNNTs have been theoretically investigated in many works. Slightly different results were presented, all of which indicated a very high Young's modulus, but slightly smaller than CNTs. So BNNTs can be widely used as a structural reinforcement of matrix materials (Zhi et al., 2010). 
First-principles, tight-binding, density functional and classical molecular mechanics approaches have been performed to characterize properties of BNNTs. Young's modulus of multi-walled boron nitride nanotubes (MWBNNTs) was obtained $1.22 \quad 0.24 \mathrm{TPa}$ using thermal vibration amplitude analysis (Chopra and Zettl, 1998). Many researchers employed the tight-binding method for calculating the axial Young's moduli of zigzag and armchair BNNTs (Verma et al., 2007). It is also observed that zigzag nanotubes have a higher Young's modulus than armchair ones. In another study, Akdim et al. indicated that Young's modulus of BNNTs varied in the range of $0.71 \sim 0.83 \mathrm{TPa}$ and was slightly dependent on the tube diameter (Akdim et al., 2003).

Using ab-initio calculations based on the density functional theory (DFT), Young's modulus of DWBNNT was calculated and the estimated values for $(2,2)$ and $(7,7),(2,2)$ and $(9,9)$ were 821 and $764 \mathrm{GPa}$, respectively (Fakhrabad and Shahtahmassebi, 2013). Also, Young's modulus of SWBNNTs with vacancy and functionalization defects was calculated by Griebel et al. (2009) using molecular dynamics (MD) simulation. They found that Young's modulus decreased with increasing defect concentration.

Young's modulus of BNNTs was reported to be 1.1-1.3 TPa from an experimental test (Bettinger et al., 2002). In another experimental effort, Young's modulus of MWBNNT was obtained $895 \mathrm{GPa}$ (Wei et al., 2010). Suryavanshi et al. (2004) applied the electric-field-induced resonance method and specified Young's modulus as $0.8 \mathrm{TPa}$.

Polymer nanocomposite combining polymers and nano-filler components have attracted research attention from the academic and industrial communities due to their diverse functional applications, good processing and relatively low cost (Mohammadimehr and Mahmudian-Najafabadi, 2013). It is reported that nano-fillers such as particles and platelets can change the crack propagation direction and consequently stop this (Rozenberg and Tenne, 2008). Crack deflection as a result of nano-sized reinforcements in a matrix has been reported to have a significant role in toughening (Sun et al., 2009). Nano-fillers can stop crack propagation along the original direction and also result in branching if agglomeration is minimized (Rozenberg and Tenne, 2008).

Lee et al. (2013) investigated the boron nitride nanoflake (BNNF) modification on epoxy resin. It was noted that strength of epoxy resin increased while Young's modulus did not significantly change. The highest strength increase was obtained at $0.3 \mathrm{wt} . \% \mathrm{BN}$ content while the highest toughness increase was achieved with $0.5 \mathrm{wt} . \% \mathrm{BN}$ content. In another work, Ulus et al. (2014) produced and investigated mechanical properties of boron nitride nanoplatelets (BNNP)-multiwall carbon nanotubes/epoxy hybrid nanocomposites. Young's modulus and tensile strength values were obtained via tensile tests. It is seen that tensile strength of epoxy resin increased from $60 \mathrm{MPa}$ to $75 \mathrm{MPa}$ (25\% increases) at $0.5 \mathrm{wt} . \%$ BNNP content.

Applications of boron nitride nanotubes/epoxy nanocomposites to adhesive joints and composite laminates were reported by Jakubinek et al. (2016). Nanocomposites containing up to $7 \mathrm{wt} . \%$ BNNTs were fabricated by planetary mixing. The effects of BNNT loading on viscosity, tensile properties and fracture toughness were determined. The elastic modulus of nanocomposite increased progressively with the BNNT loading up to $5 \mathrm{wt} . \%$. While ultimate strain only decreased with BNNT addition, the fracture toughness also reached a maximum around 5 wt.\%.

Ghorbanpour Arani et al. (2012a) analyzed the electro-thermo-elastic stress of a piezoelectric polymeric thick-walled cylinder reinforced by BNNTs. They also investigated the electro-thermo-mechanical axial buckling behavior of a piezoelectric polymeric cylindrical shell reinforced with a double-walled boron-nitride nanotube using the principle of minimum total potential energy approach in conjunction with the Rayleigh-Ritz method (Ghorbanpour Arani et al., 2012b). Bending and free vibration of a nonlocal functionally graded nanocomposite Timoshenko beam model reinforced by SWBNNT were reported based on a modified coupled stress theory (Davar and Sadri, 2016). Also, the effects of BNNTs on the elastic modulus of beta tricalcium phosphate and hydroxyapatite were analyzed using a RVE model. The predicted elastic moduli of the 
$\beta$-TCP-BNNTs and HA-BNNTs composites showed $24.1 \%$ and $26.3 \%$ enhancement, respectively (Davar and Sadri, 2017). They also investigated the effect of BNNTs on the stress-intensity factor $\left(K_{I}\right)$ of a semi-elliptical surface crack in a wide range of matrices using a finite element model. The results showed that a higher mismatch difference between the elastic modulus of the matrix and BNNTs resulted in further reduction in $K_{I}$ value (Mortazavi et al., 2013).

Experimental studies on nanocomposites are time consuming, costly, and require accurate implementation. Therefore, analytical, computational and theoretical approaches are attractive methods of predicting mechanical properties of composites. Researchers usually employ a small part of the whole composite, which is called the unit cell or RVE to avoid expensive and enormous computational calculations (Gojny et al., 2005).

The experimental studies on nanocomposites are time-consuming, costly, and require accurate implementation, so the analytical, computational and theoretical approaches are attractive methods for nanocomposite simulation and predicting mechanical properties. In this work, a representative volume element (RVE) of epoxy/BNNT nanocomposites based on multi-scale modeling is considered.

Mechanical behaviors of BNNTs is studied using a three-dimensional finite element (FE) model, named as the space frame model. Ansari et al. (2015) used DFT calculations to obtain exact force constants of BNNT which are employed in determining the element properties. Fundamental to these approaches, BNNTs are considered as geometric space frame structures and can be analyzed by classical structural mechanics. In this paper, three-dimensional RVEs with different chirality of BNNT are simulated and analyzed in three fracture modes. In all fracture modes, the stress intensity factor of nanocomposites is determined and compared with the pure matrix one.

\section{Multi-scale modeling}

In fracture mechanics based on the crack surfaces displacement, three crack modes of the opening mode (tensile mode), shearing mode (sliding mode), tearing mode (out-of-plane) are considered. Stress intensity factors of RVEs are determined using the $J$ integral technique with ANSYS Parametric Design Language (APDL) based on finite element analysis. Similar loads and boundary conditions are applied to both the neat matrix and nanocomposite in the three fracture modes, and then their stress intensity factors are determined and compared together.

In the bottom-up analysis method, one firstly obtains the effective material constants using a low-scale such as the nano, meso (Tserpes et al., 2008) or micro RVE model (Gibson et al., 2007), then applies it in the high-scale FE simulation in which the material is assumed to be equivalently homogeneous according to the theory of continuum mechanics. In the top-down or global-local method, one firstly finds the properties in the local region of a macro-scale sample under practical loads, then applies the resultant loads or displacements to the boundary of a smaller region as a new input.

\subsection{Nanotube}

BNNTs atoms are bonded together with covalent bonds forming a hexagonal lattice. The displacement of individual atoms under an external force is constrained by the bonds. Therefore, the total deformation of the nanotube is a result of the interactions between the bonds. By considering the bonds as connecting load-carrying elements, and the atoms as joints of the connecting elements, BNNTs may be simulated as space-frame structures (Ansari et al., 2015).

The 3D FE model is developed using the ANSYS commercial FE code. The 3D elastic BEAM4 element is used for modeling the bonds. The properties of these elements are obtained by a linkage between the potential energy of bonds (from a chemical point of view) and the strain 
energy of mechanical elements (from a mechanical point of view). To represent the covalent bond between boron and nitrogen atoms, a circular beam of length $l$, diameter $d$, Young's modulus $E$, and shear modulus $G$ is considered (Ansari et al., 2015). The required properties of the beam element are given in Table 1.

Table 1. The properties of beam elements for real BNNT (Ansari et al., 2015)

\begin{tabular}{|l|c|}
\hline Diameter $d$ & $1.648 \AA$ \\
\hline Cross-sectional area $A$ & $2.132 \AA^{2}$ \\
\hline Boron-nitrogen bond & $1.45 \AA$ \\
\hline Polar inertia momentum $I_{x x}$ & $0.7250 \AA^{4}$ \\
\hline Inertia momentum $I_{z z}=I_{y y}=I$ & $0.3625 \AA^{4}$ \\
\hline Young's modulus $E$ & $4.2155 \cdot 10^{-8} \mathrm{~N} / \AA^{2}$ \\
\hline Shear modulus $G$ & $4.9437 \cdot 10^{-9} \mathrm{~N} / \AA^{2}$ \\
\hline
\end{tabular}

A routine code has been created using the ANSYS macro language, for automatic generation of FE models. The thickness of BNNT is considered as $0.34 \mathrm{~nm}$ and also, the center of the BNNT wall is placed at the midsection of the tube thickness. The FE meshes, loading and boundary conditions of $(10,10)$ BNNT with length of $80 \AA$ are shown in Fig. 1. Young's and shear modulus of BNNT are found by tension and torsion loading. They are in ranges of 1.039-1.041 TPa and 0.44-0.52 TPa, respectively. The experimental and theoretical elastic moduli of BNNT are given in Table 2. The current results are in good agreement with the simulation and experimental values.
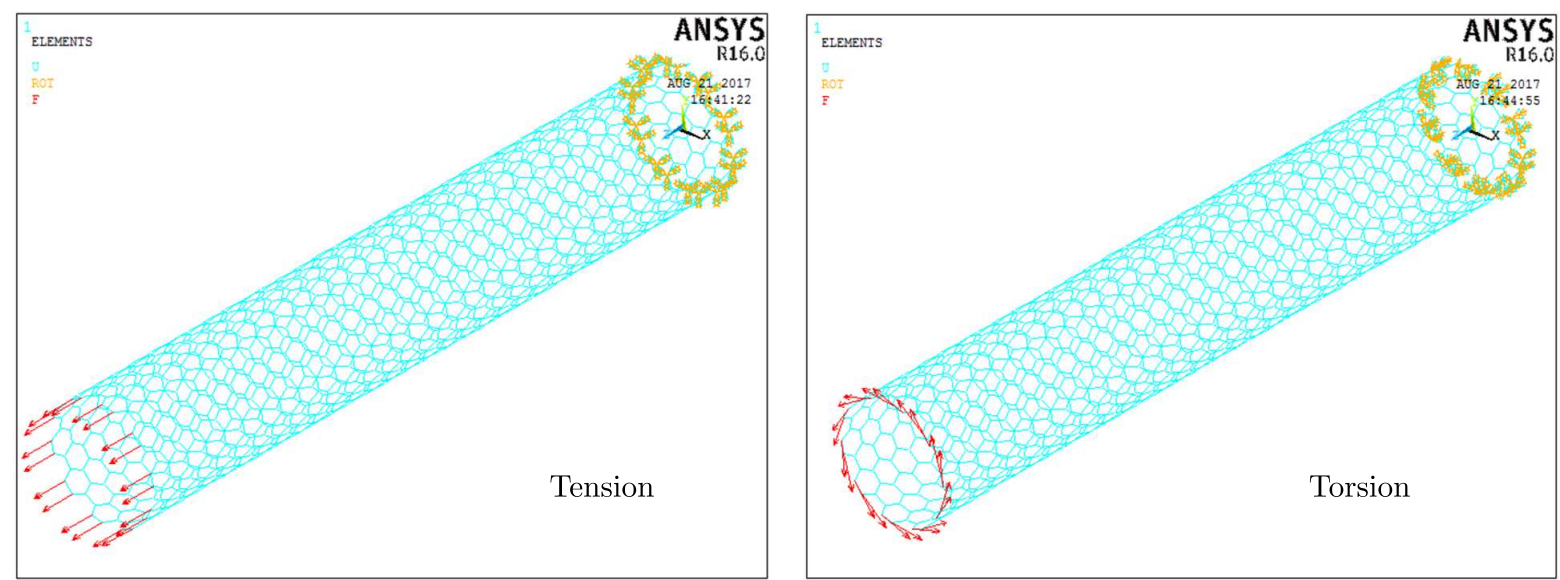

Fig. 1. FE meshes of BNNT $(10,10)$ with loading and boundary conditions

\subsection{Inter-phase between nanotube and polymer}

The bonding between the embedded BNNT and its surrounding polymer takes place through $\mathrm{vdW}$ and electrostatic interactions in the absence of chemical functionalization. Since vdW contributes more significantly by three higher orders of magnitude than electrostatic energy, the electrostatic interactions can be neglected in comparison with vdW interactions (Gou et al., 2004). So, only vdW interactions are considered between the BNNT and the matrix. The $\mathrm{vdW}$ forces are most often modeled using the famous Lennard-Jones equation (Battezzatti et al., 1975)

$$
F_{V d W}=4 \frac{\varepsilon}{r}\left[-12\left(\frac{\sigma}{r}\right)^{12}+6\left(\frac{\sigma}{r}\right)^{6}\right]
$$


Table 2. Elastic moduli from BNNT of simulation and experimental works

\begin{tabular}{|c|c|c|}
\hline Elastic moduli & {$[\mathrm{TPa}]$} & Reference \\
\hline \hline \multirow{5}{*}{ Young's modulus } & $1.22 \pm 0.24$ & Chopra and Zettl $(1998)$ \\
\cline { 2 - 3 } & $1.022-1.112$ & Fereidoon et al. $(2015)$ \\
\cline { 2 - 3 } & $0.862-0.94$ & Verma et al. $(2007)$ \\
\cline { 2 - 3 } & $0.71-0.83$ & Akdim et al. $(2003)$ \\
\cline { 2 - 3 } & 0.895 & Fakhrabad and Shahtahmassebi $(2013)$ \\
\cline { 2 - 3 } & $0.7-1.2$ & Gerieble et al. $(2009)$ \\
\cline { 2 - 3 } & $0.7-1.2$ & Bettinger et al. $(2002)$ \\
\cline { 2 - 3 } & $1.1-1.3$ & Wei et al. $(2010)$ \\
\cline { 2 - 3 } & $0.764-0.821$ & Suryavanshi et al. $(2004)$ \\
\cline { 2 - 3 } & 1 & Chowdhury et al. $(2010)$ \\
\cline { 2 - 3 } & $1.039-1.041$ & Current work \\
\hline Shear modulus & 0.42 & Chowdhury et al., 2010 \\
\cline { 2 - 3 } & $0.44-0.52$ & Current work \\
\hline
\end{tabular}

where $r$ is the separation distance between the pair of atoms, $\varepsilon$ is the bond energy at the equilibrium distance, and $\sigma$ is the van der Waals separation distance. The equilibrium distance between atoms is $\sqrt[6]{2} \sigma$. By introducing $x$ as the distance from the equilibrium distance, the Lennard-Jones force is represented in Eq. $(2.2)_{2}$

$$
\begin{aligned}
& x=r-\sqrt[6]{2} \sigma \\
& F(X)=-24 \frac{\varepsilon}{\sigma}\left[2\left(\frac{\sigma}{x+\sqrt[6]{2} \sigma}\right)^{13}-\left(\frac{\sigma}{x+\sqrt[6]{2} \sigma}\right)^{7}\right] \\
& \sigma=\frac{\sigma_{n}+\sigma_{m}}{2} \quad \varepsilon=\sqrt{\varepsilon_{n} \varepsilon_{m}}
\end{aligned}
$$

where $m$ and $n$ sub-indexes denote the matrix and nanotube, respectively. The Lennard-Jones potential parameters $(\varepsilon$ and $\sigma$ ) of the materials are given in Table 3. BNNT is a synthase from boron and nitrogen atoms, therefore the Lennard-Jones potential parameters of BNNT are approximately considered the average of boron and nitrogen parameter values. Also, these values are represented in Table 3. The Lennard-Jones potential parameters for van der Waals interaction between the BNNT and epoxy are determined as $\sigma=3.897 \AA$ and $\varepsilon=0.00297 \mathrm{nNnm}$ by replacing $L-J$ parameters of BNNT and epoxy. Also, the equilibrium distance between matrix and nanotube is $0.4374 \mathrm{~nm}$.

Table 3. Lennard-Jones potential parameters of the materials

\begin{tabular}{|l|c|c|c|c|}
\hline Materials & $\sigma[\AA]$ & Reference & $\varepsilon$ & Reference \\
\hline \hline Nitrogen & 3.365 & $\begin{array}{c}\text { Chen } \text { et al. }(2015) \\
\text { Zhang and Wang (2016) }\end{array}$ & $6.281 \mathrm{meV}$ & $\begin{array}{c}\text { Chen } \text { et al. }(2015) \\
\text { Zhang and Wang (2016) }\end{array}$ \\
\hline Boron & 3.453 & $\begin{array}{c}\text { Chen } \text { et al. }(2015) \\
\text { Zhang and Wang (2016) }\end{array}$ & $4.16 \mathrm{meV}$ & $\begin{array}{c}\text { Chen } \text { et al. }(2015) \\
\text { Zhang and Wang (2016) }\end{array}$ \\
\hline BNNT & 3.409 & - & $\begin{array}{c}5.2205 \mathrm{meV}- \\
0.00083642 \mathrm{nNnm}\end{array}$ & - \\
\hline Epoxy & 4.383 & Yang et al. $(2014)$ & $\begin{array}{c}1.519 \mathrm{kcal} / \mathrm{mol}- \\
-0.1055 \mathrm{nNnm}\end{array}$ & Gou et al. (2004) \\
\hline
\end{tabular}

The vdW interactions between the BNNT and the inner surface nodes of the surrounding resin are modeled using a 3D non-linear spring element based on the corresponding data of the force-displacement curve (Hemmatian et al., 2012). COMBIN39 element is used for this purpose 
and the parameters are adjusted to obtain a translational spring. A macro is written to create elements between the BNNT and the inner surface of the surrounding resin nodes that their distance is lower than $0.7 \mathrm{~nm}$.

\subsection{Matrix}

SOLID45 elements are utilized for modeling of the matrix. This element is used for the 3D modeling of solid structures. SOLID45 is defined by eight nodes having three degrees of freedom at each node: translations in the nodal $x, y$ and $z$ directions. Young's modulus of the epoxy matrix is considered as $2.9 \mathrm{GPa}$ (Fereidoon et al., 2013). Nanocomposites consisting of $\% 5$ volume fraction of BNNT with length of $80 \AA$ are simulated. The FE meshes of RVE used for crack analysis are shown in Fig. 2. The elements of the crack tip are refined to increase the accuracy of analysis as represented in Fig. 2.
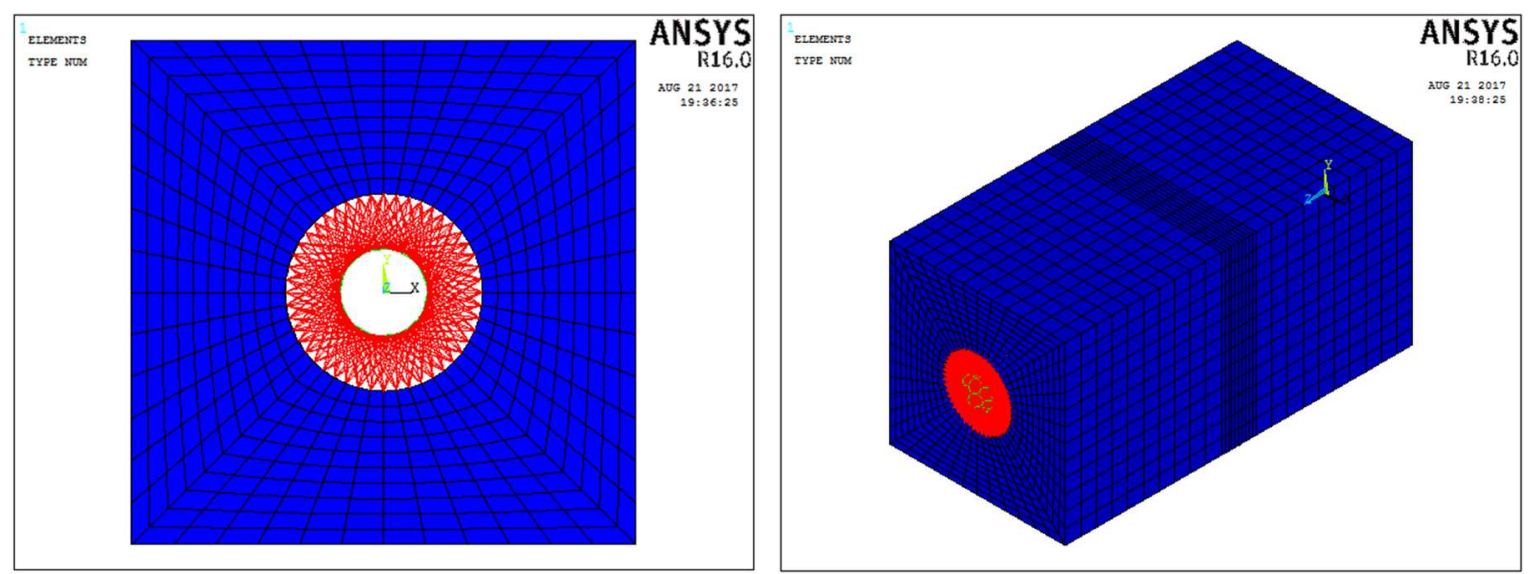

Fig. 2. The FE meshes of nanocomposite RVE

Stress intensity factors of the neat matrix and nanocomposite are compared for similar loading and boundary conditions. The loading and boundary conditions of three fracture modes including the opening, shearing and tearing of the nanocomposite with 5 Vo.\% $(5,5)$ BNNT are shown in Fig. 3. In order to apply the conditions of the opening mode, the middle nodes of the RVE are fully built-in (zero displacement and rotation conditions), while the nodes of two ends are subjected to tensile forces. In shearing and tearing modes, the nodes of the back of the RVE are fully built-in (zero displacement and rotation conditions), while the middle nodes are constrained in the $Z$ direction. Shearing and tearing forces are applied to the front nodes of these RVE's.
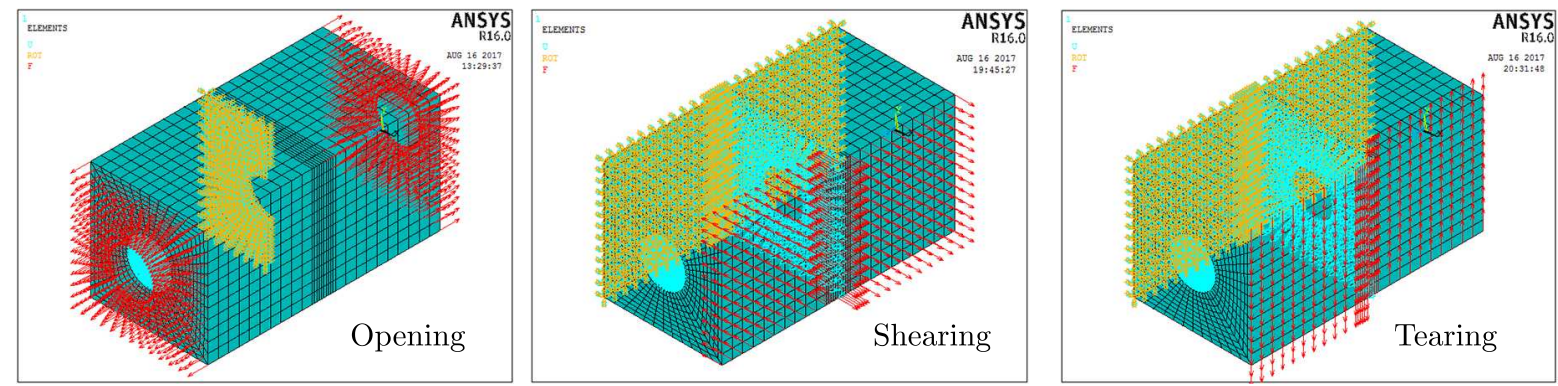

Fig. 3. Loadings and boundary conditions of RVE for three modes 

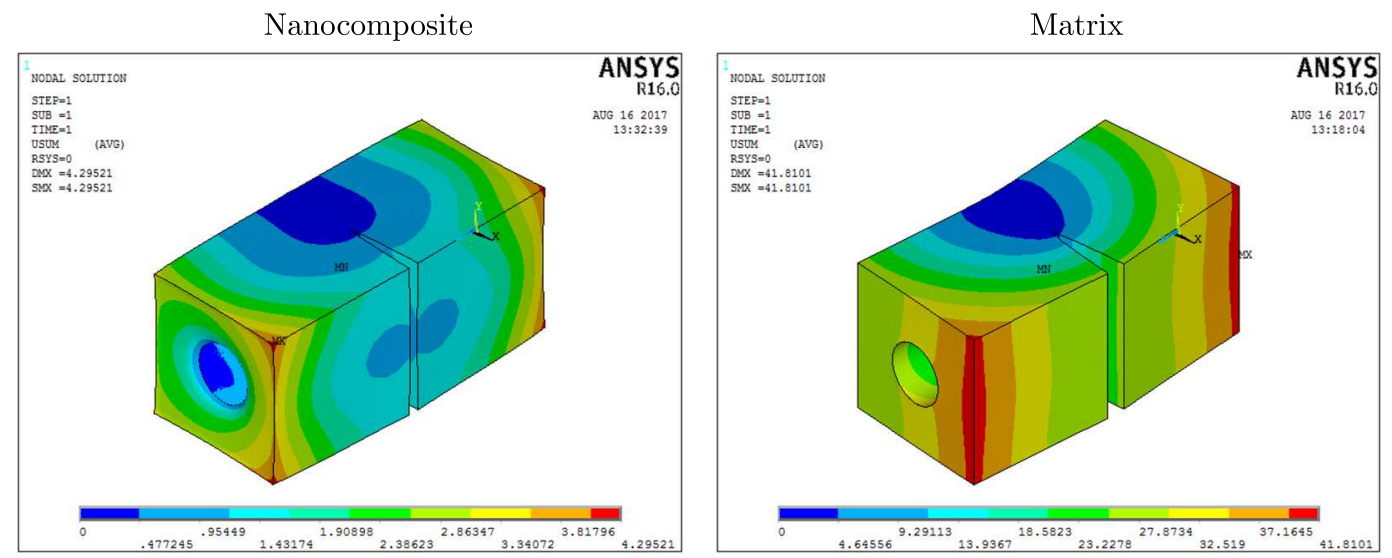

Chirality
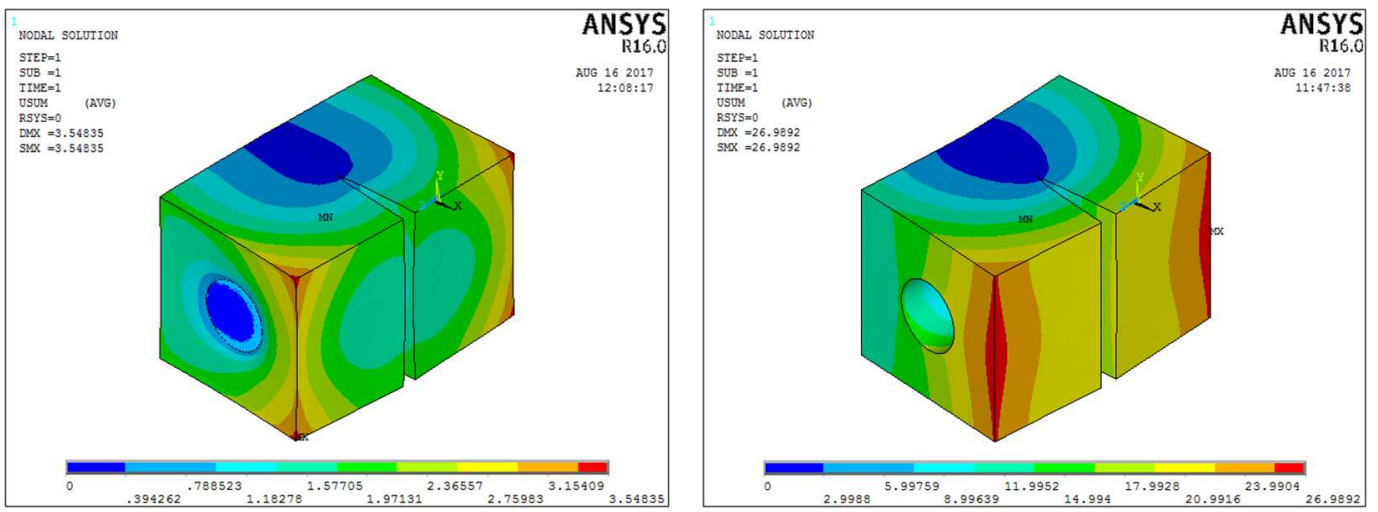

$(5,5)$
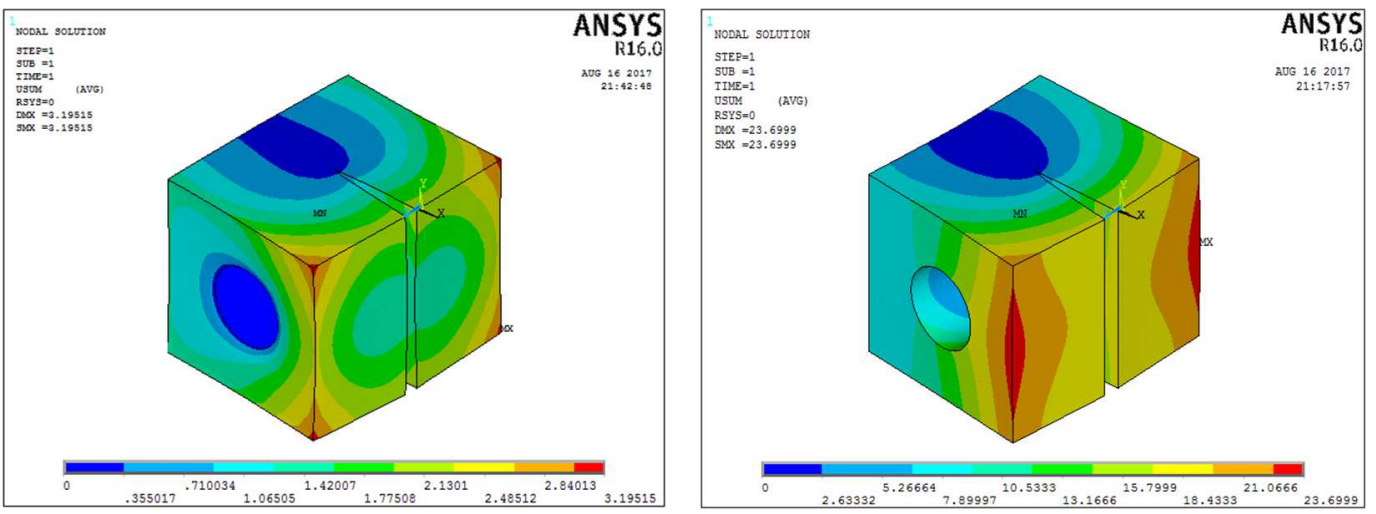

$(10,10)$
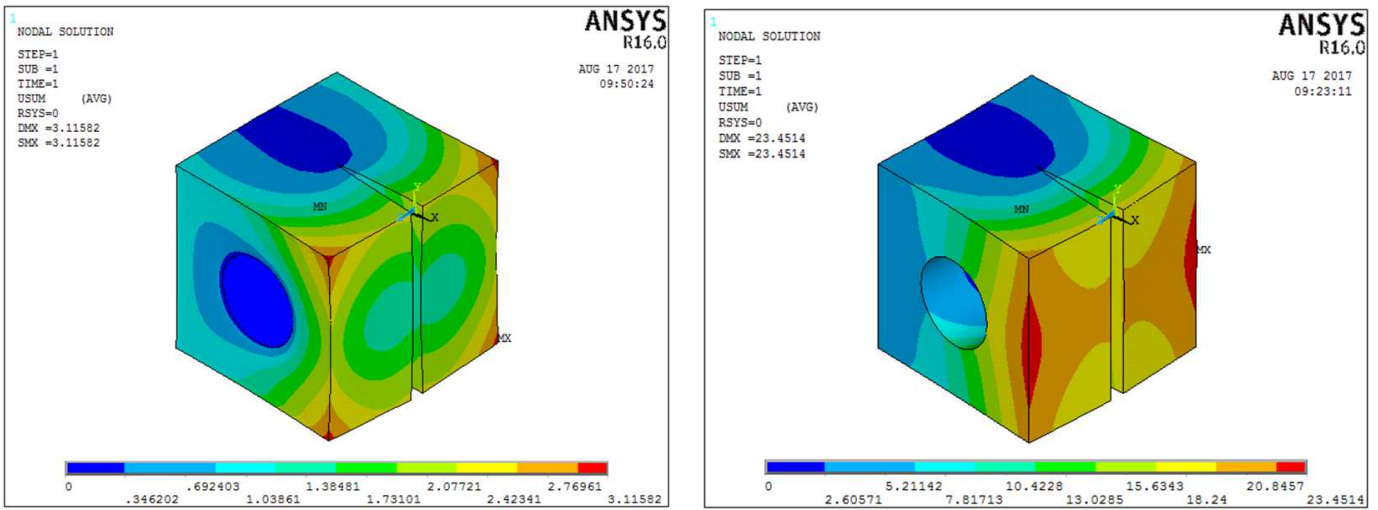

$(15,15)$

$(20,20)$

Fig. 4. Displacement $[\AA]$ contours of the opening mode: neat matrix and nanocomposite 

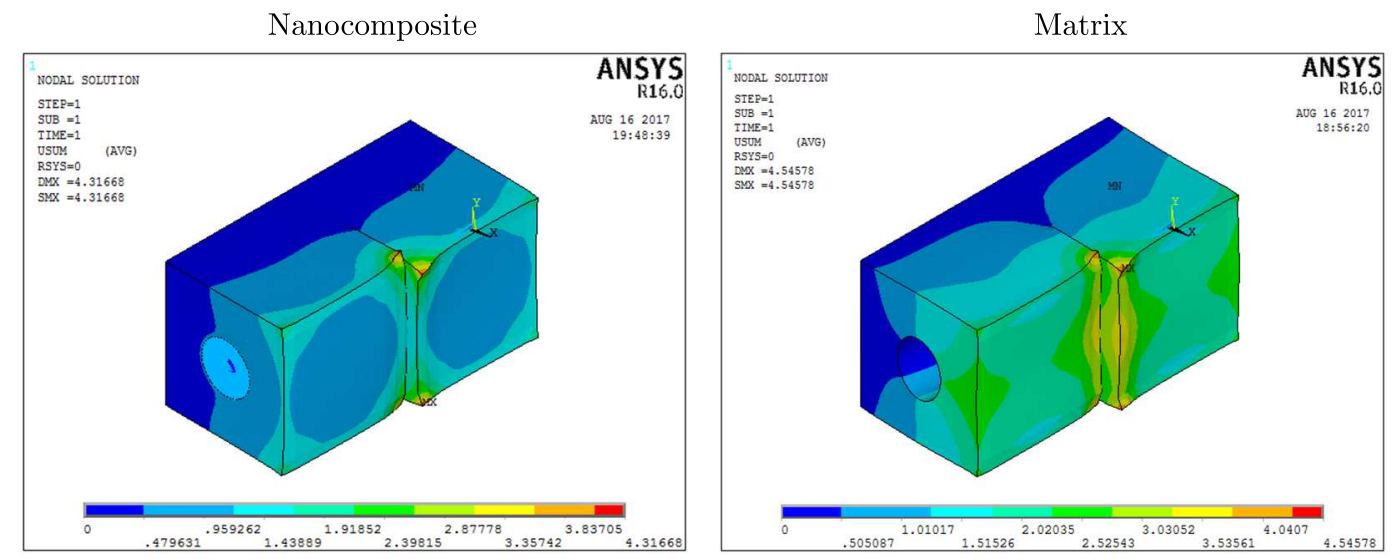

Chirality
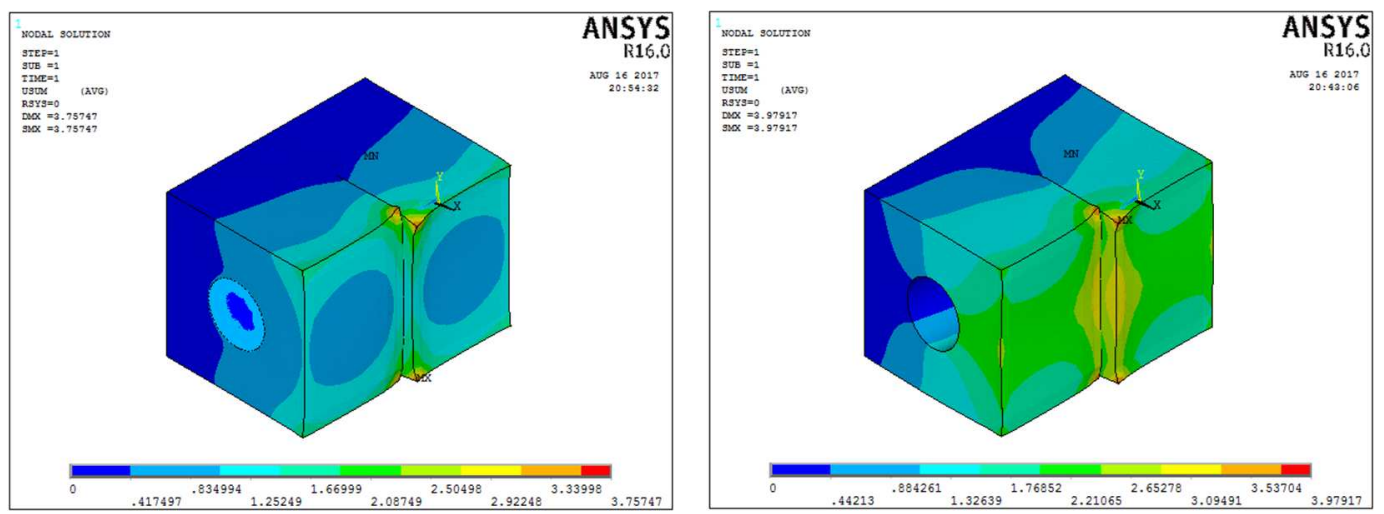

$(5,5)$
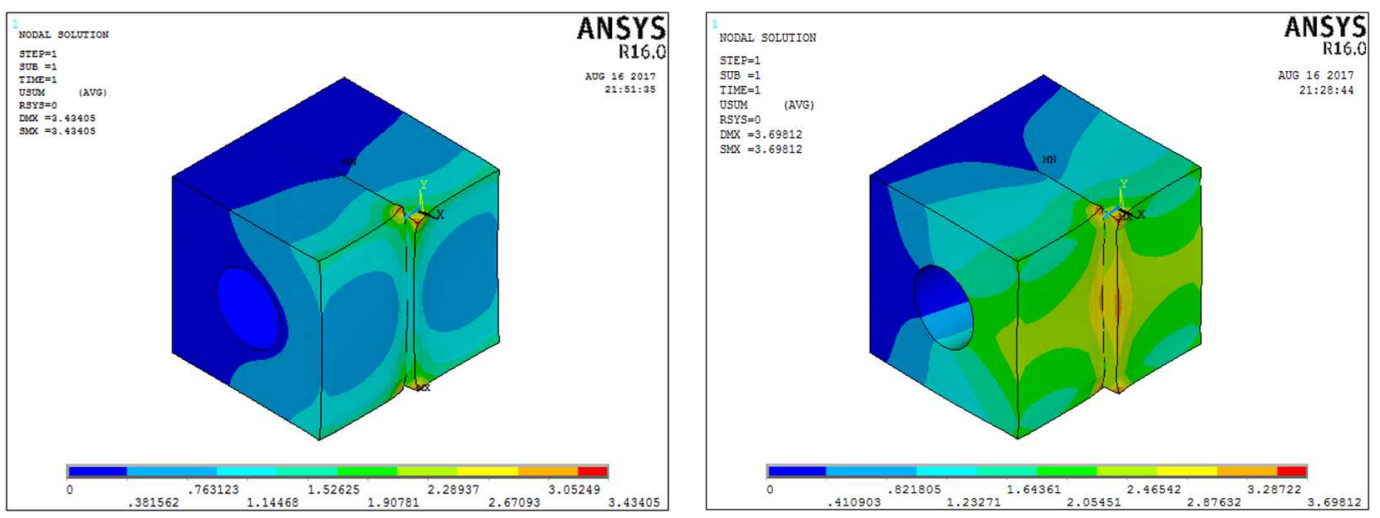

$(10,10)$
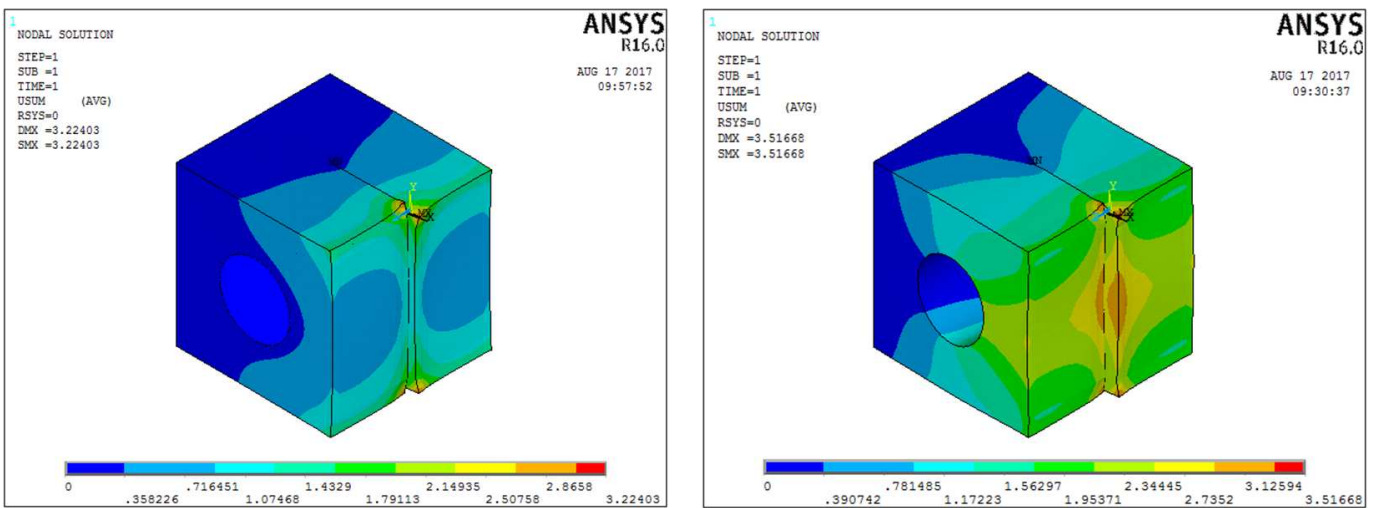

$(15,15)$

$(20,20)$

Fig. 5. Displacement $[\AA]$ contours of the shearing mode: neat matrix and nanocomposite 

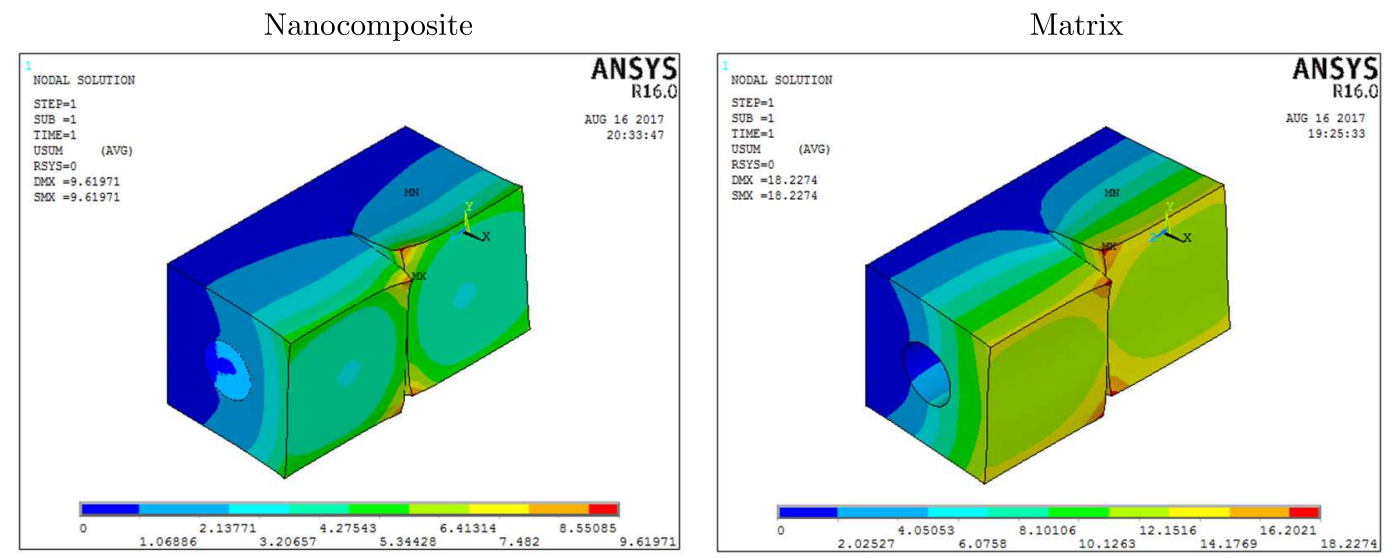

Chirality
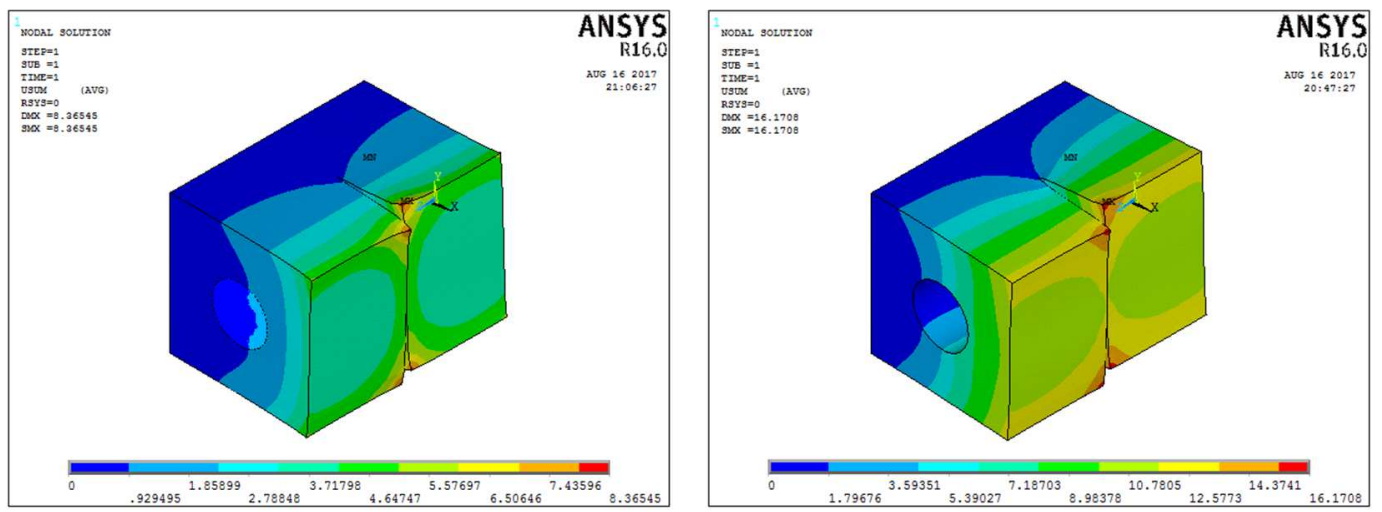

$(5,5)$
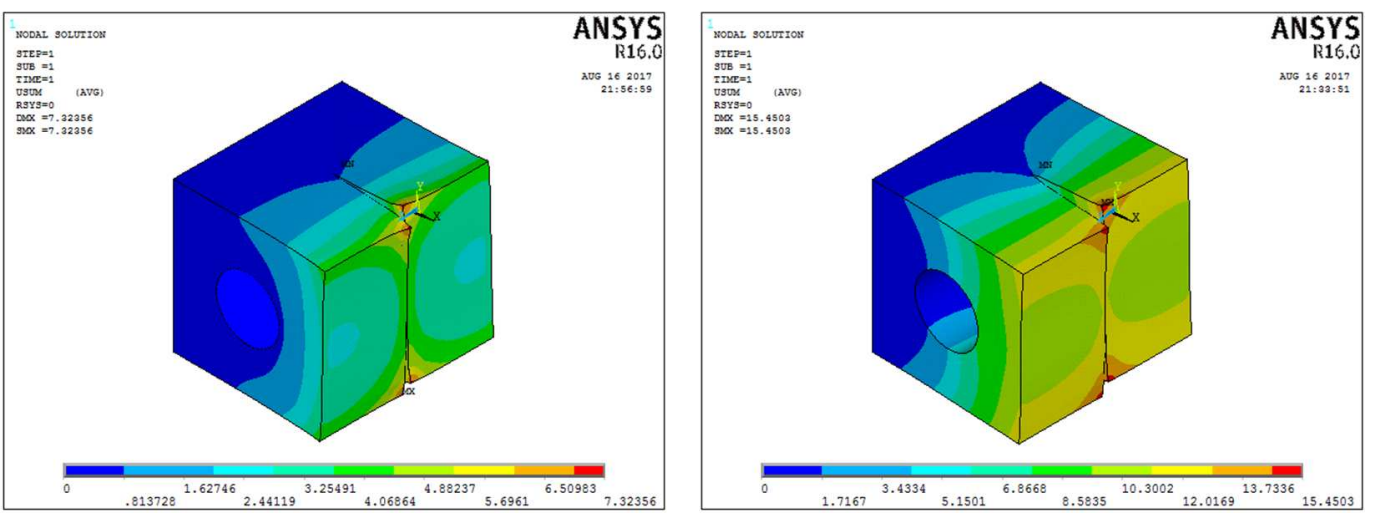

$(10,10)$
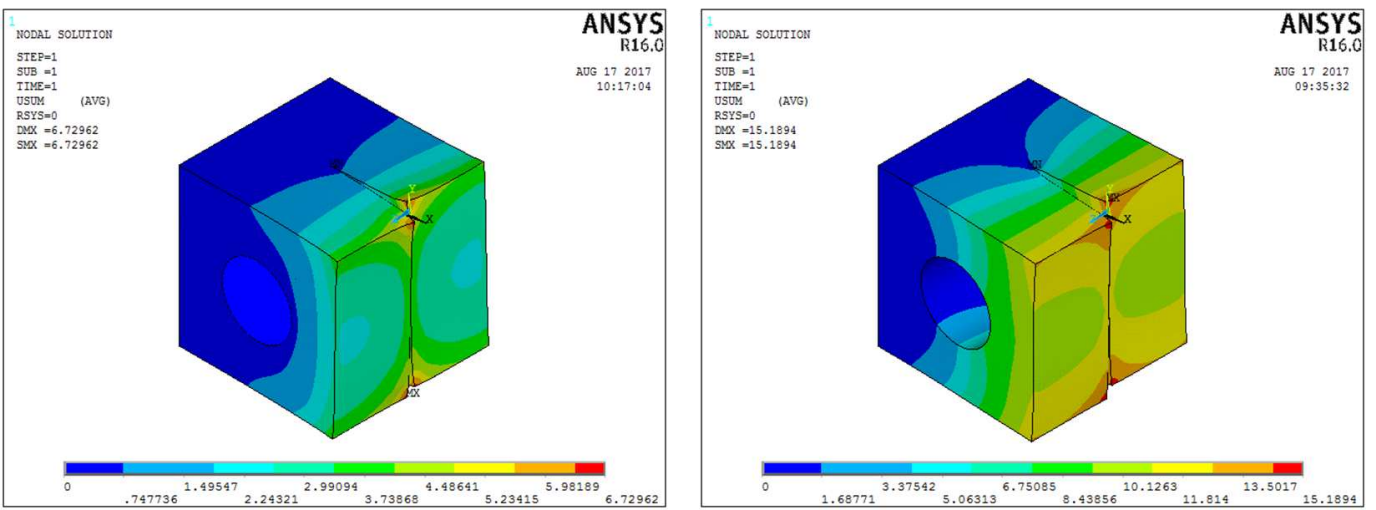

$(15,15)$

$(20,20)$

Fig. 6. Displacement $[\AA]$ contours of the tearing mode: neat matrix and nanocomposite 


\section{Results and discussion}

In this study, fracture analysis of an epoxy/BNNT nanocomposite reinforced with four chirality $(5,5),(10,10),(15,15)$ and $(20,20)$ are implemented. It is observed that when nanotubes are vertical to the crack path, the minimum stress intensity factor and the maximum effect on crack resistance are achieved. In this condition, while the crack is in the middle of RVE (bridging condition), this effect is stronger. Nanotubes with constant length and different chirality have been used for the bridging condition.

Displacement contours of the neat matrix and nanocomposite in the opening, shearing and tearing modes are shown in Figs. 4, 5 and 6, respectively. The dimension of displacement is Angstrom.

The maximum displacement of RVE is decreased by adding BNNT, and this phenomenon in the opening mode is evident. Diagrams of normalized stress intensity factors (ratio of the nanocomposite stress intensity factor to that of the neat matrix) of the fracture modes are plotted against chirality in Fig. 7.

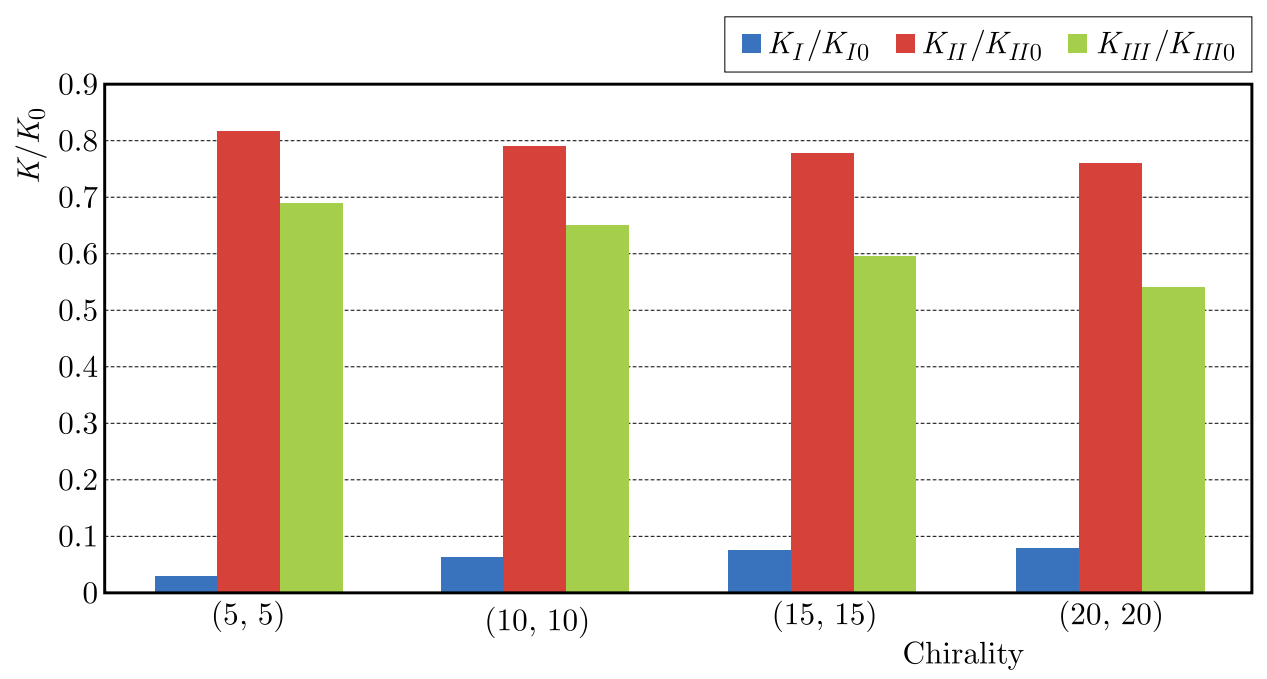

Fig. 7. Normalized stress intensity factors against chirality

The results indicate that with the addition of BNNT to epoxy, the stress intensity factors of three modes decrease. Also, by increasing the chirality and consequently, BNNT diameter, the crack resistance of shearing and tearing modes enhances, and the stress intensity factor of the opening mode reduces. Boron nitride nanotube bridges the crack path and resists against crack propagation. On the other hand, the bridging arises in all three modes, and the stress intensity factor decreases. This phenomenon is reported in experimental research about CNT too (Mirjalili and Hubert, 2010). Therefore, adding the BNNT to the matrix improves the crack resistance, which is considerable in the opening and tearing modes.

In modeling and simulation, the chirality, length, diameter, and weight percentage of the nanotube are important. But in the experimental method, the weight percentage and range of length and diameter is considered. Hence, appropriate verification between the results of experimental and simulation is difficult. Also, based on literature review, the experimental and simulation results do not deal with fracture behavior of BNNT/epoxy in the three modes. Multiscale simulations from nano to macro, or reversely, improve the specification of toughening mechanisms. 


\section{Conclusions}

A three-dimensional FEM of BNNTs has been proposed. Nodes are placed at locations of boron and nitrogen atoms, and bonds are modeled using three-dimensional elastic beam elements by considering a linkage between molecular and continuum mechanics. The simulation performed under minimal computational time by requiring minimal computational power. The determined elastic moduli of BNNTs are in a good agreement with the real parameters. A three-dimensional study of three fracture modes in epoxy/BNNT has been performed based on a multi-scale method. Van der Waals bonds between the resin and nanotube are simulated by non-linear spring elements based on the Lennard-Jones potential.

Stress intensity factors of three fracture modes have been computed by $J$ integral. The effect of BNNT on the stress intensity factor of nanocomposites and crack propagation is investigated. The results indicated that BNNTs have a significant effect on preventing crack propagation. Also, by adding a nanotube, stress intensity factors decreased and, consequently, the crack resistance increased. The results indicated that the crack resistance improved by increasing the chirality (radius). Finally, by adding BNNT to the matrix, the improvement of matrix fracture properties is evident.

\section{References}

1. Akdim B., Achter R.P., Duan X.F., Adams W.W., 2003, Comparative theoretical study of single-wall carbon and boron-nitride nanotubes, Physical Review B, 67, 245404

2. Ansari R., Rouhi S., Mirnezhad M., Aryayi M., 2015, Stability characteristics of single-walled boron nitride nanotubes, Archives of Civil and Mechanical Engineering, 15, 162-170

3. Battezzatti L., Pisani C., Ricca F., 1975, Equilibrium conformation and surface motion of hydrocarbon molecules physisorbed on graphite, Journal of the Chemical Society, 71, 1629-1639

4. Bettinger H.F., Dumitric T.T., Scuseria G.E., Yakobson B.I., 2002, Mechanically induced defects and strength of BN nanotubes, Physical Review B, 65, 041406

5. Chang C.W., Han W.Q., Zettl A., 2005, Thermal conductivity of B-C-N and BN nanotubes, Applied Physics Letters, 86, 173102

6. Chen X., Zhang L., Park C., Fay C.C., Wang X., Ke C., 2015, Mechanical strength of boron nitride nanotube-polymer interfaces, Applied Physics Letters, 107, 253105

7. Chen Y., Zou J., Campbell S.J., Caer G.L., 2004, Boron nitride nanotubes: pronounced resistance to oxidation, Applied Physics Letters, 84, 2430-2432

8. Chopra N.G., Luyken R.J., Cherrey K., Crespi V.H., Cohen M.L., Louie S.G., Zettl A., 1995, Boron nitride nanotubes, Science, 269, 966-967

9. Chopra N.G., Zettl A., 1998, Measurement of the elastic modulus of a multi-wall boron nitride nanotube, Solid State Communications, 105, 297-300

10. Chowdhury R., Wang C.Y., Adhikari S., Scarpa F., 2010, Vibration and symmetry-breaking of boron nitride nanotubes, Nanotechnology, 21, 365702

11. Davar A., Sadri S., 2016, Finite element analysis of the effect of boron nitride nanotubes in beta tricalcium phosphate and hydroxyapatite elastic modulus using the RVE model, Composites Part B: Engineering, 90, 336-340

12. Davar A., Sadri S., 2017, Finite element analysis of boron nitride nanotubes' shielding effect on the stress intensity factor of semielliptical surface crack in a wide range of matrixes using RVE model, Composites Part B: Engineering, 110, 351-360

13. Fakhrabad D.V., Shahtahmassebi N., 2013, First-principles calculations of the Young's modulus of double wall boron-nitride nanotubes, Materials Chemistry and Physics, 138, 2, 963-966 
14. Fereidoon A., Mostafaei M., Ganji M.D., Memarian F., 2015, Atomistic simulations on the influence of diameter, number of walls, interlayer distance and temperature on the mechanical properties of BNNTs, Superlattices and Microstructures, 86, 126-133

15. Fereidoon A., Rajabpour M., Hemmatian H., 2013, Fracture analysis of epoxy/SWCNT nanocomposite based on global-local finite element model, Composites: Part B, 54, 400-408

16. Ghorbanpour Arani A., Haghshenas A., Amir S., Azami M., Khoddami Maraghi Z., 2012a, Electro-thermo-mechanical response of thick-walled piezoelectric cylinder reinforced by BNNTs, Journal of Nanostructures, 2, 113-124

17. Ghorbanpour Arani A., Shams S., Amir S., Khoddami Maraghi Z., 2012b, Effects of electro-thermal fields on buckling of a piezoelectric polymeric shell reinforced with DWBNNTs, Journal of Nanostructures, 2, 345-355

18. Gibson R., 2007, Principles of Composite Material Mechanics, CRC Press

19. Gojny F.H., Wichmann M.H.G., Fiedler B., Schulte K., 2005, Influence of different carbon nanotubes on the mechanical properties of epoxy matrix composites - a comparative study, Composites Science and Technology, 65, 2300-2313

20. Gou J., Minaei B., Wang B., Liang Z., Zhang C., 2004, Computational and experimental study of interfacial bonding of single-walled nanotube reinforced composites, Computational Materials Science, 31, 225-236

21. Griebel M., Hamaekers J., Heber F., 2009, A molecular dynamics study on the impact of defects and functionalization on the Young modulus of boron-nitride nanotubes, Computational Materials Science, 45, 4, 1097-1103

22. Hemmatian H., Fereidoon A., Rajabpour M., 2012, Investigation of crack resistance in single walled carbon nanotube reinforced polymer composites based on FEM, Journal of Ultrafine Grained and Nanostructured Materials, 45, 13-18

23. Jakubinek M.B., Martinez-Rubi Y., Ashrafi B., Yourdkhani M., Rahmat M., Djokic D., Guan J., Su Kim K., Kingston C.T., Simard B., Johnston A., 2016, Nanoreinforced epoxy composites based on boron nitride nanotubes and their application to adhesive joints and composite laminates, Proceedings of 3rd Annual Composites and Advanced Materials Expo, CAMX 2016, Anaheim, United States

24. Khaleghian M., Azarakhshi F., 2016, Electronic properties studies of Benzene under boron nitride nano ring field, International Journal of Nano Dimension, 7, 290-294

25. Lee D., Song S.H., Hwang J., Jin S.H., Park K.H., Kim B.H., Hong S.H., Jeon S., 2013, Enhanced mechanical properties of epoxy nanocomposites by mixing noncovalently functionalized boron nitride nanoflakes, Small, 9, 2602-2610

26. Mirjalili V., Hubert P., 2010, Modelling of the carbon nanotube bridging effect on the toughening of polymers and experimental verification, Composites Science and Technology, 70, 1537-1543

27. Mohammadimehr M., Mahmudian-Najafabadi M., 2013, Bending and free vibration analysis of nonlocal functionally graded nanocomposite Timoshenko beam model reinforced by SWBNNT based on modified coupled stress theory, Journal of Nanostructures, 3, 483-492

28. Molani F., 2017, The effect of C, Si, N, and P impurities on structural and electronic properties of armchair boron nanotube, Journal of Nanostructure in Chemistry, 7, 243-248

29. Mortazavi B., Baniassadi M., Bardon J., Ahzi S., 2013, Modeling of two-phase random composite materials by finite element, Mori-Tanaka and strong contrast methods, Composites Part B, Engineering, 45, 1117-1125

30. Rozenberg B.A., Tenne R., 2008, Polymer-assisted fabrication of nanoparticles andnanocomposites, Progress in Polymer Science, 33, 40-112

31. Sun L., R. Gibson F., Gordaninejad F., Suhr J., 2009, Energy absorption capability of nanocomposites: a review, Composites Science and Technology, 69, 2392-2409 
32. Suryavanshi A.P., Yu M., Wen J., Tang C., Bando Y., 2004, Elastic modulus and resonance behavior of boron nitride nanotubes, Applied Physics Letters, 84, 2527-2529

33. Tserpes K., Papanikos P., Labeas G., Pantelakis S., 2008, Multi-scale modeling of tensile behavior of carbon nanotube-reinforced composites, Theoretical and Applied Fracture Mechanics, 49, 51-60

34. Ulus H., Üstün T., Eskizeybek V., ŞAhin Ö.S., Avci A., Ekrem M., 2014, Boron nitride-MWCNT/epoxy hybrid nanocomposites: Preparation and mechanical properties, Applied Surface Science, 318, 37-42

35. Verma V., Jindal V., Dharamvir K., 2007, Elastic moduli of a boron nitride nanotube, Nanotechnology, 18, 435711

36. Wei X., Wang M.S., Bando Y., Golberg D., 2010, Tensile tests on individual multi-walled boron nitride nanotubes, Advanced Materials, 22, 43, 4895-4899

37. Yang S., Cui Z. Qu J., 2014, A coarse-grained model for epoxy molding compound, The Journal of Physical Chemistry B, 118, 1660-1669

38. ZHANG L., WANG X., 2016, DNA sequencing by hexagonal boron nitride nanopore: a computational study, Nanomaterials, 6, 111

39. Zhi C., Bando Y., Tang C., Golberg D., 2010, Boron nitride nanotubes, Materials Science and Engineering: $R, \mathbf{7 0}, 92-111$

Manuscript received April 14, 2018; accepted for print September 10, 2018 